\title{
Reliability and validity of 3 different Chinese versions of the Oxford knee score (OKS)
}

\author{
James Reeves Mbori Ngwayi ${ }^{1,2^{*}}$, Jie Tan ${ }^{1}$, Ning Liang ${ }^{1}$ and Daniel Edward Porter ${ }^{3}$
}

\begin{abstract}
Background: Different Chinese versions of the Oxford Knee Score (OKS) are available for knee arthritis assessment. These include the Malaysian, Hong Kong and Singaporean Chinese versions with slight variations in wordings and use of Cantonese in the Hong Kong Version. This study evaluated the validity and reliability of the different Chinese OKS versions in Mainland China.
\end{abstract}

Methods: One hundred ninety four China mainland-based patients participated in the study, each being diagnosed with knee osteoarthritis. The patients were randomly assigned into 3 groups: Group A completed the Malaysian OKS; Group B completed the Singaporean OKS; Group C completed the Hong Kong OKS. Participants also completed the 36-item Short Form Survey (SF 36). The electronic versions of the questionnaires completed by the patients were sent to smart devices via a social media platform.

Results: Interclass coefficients for test-retest reliability of the groups were 0.917 in group A, 0.921 in group B, 0.824 in group C. Cronbach alpha results for internal consistency of the 3 groups were: 0.912 in group A, 0.896 in group B, 0.846 in group C. Spearson correlation results with individual SF-36 domains were as follows: Group A showed strong negative correlations with bodily pain and physical function domains; group B exhibited moderate negative correlations with the bodily pain and physical function domains; group $C$ revealed strong negative correlations with the bodily pain and physical function domains and weak negative correlations with vitality domain.

Conclusions: Different Chinese versions of the OKS showed good reliability and convergent validity in mainland samples of patients with knee osteoarthritis, supporting their use in research and other related studies.

Keywords: Patient reported outcome measures, Reliability, Knee osteoarthritis

\section{Introduction}

The oxford knee score (OKS) is a commonly used patient-reported outcome measure originally designed specifically for the evaluation of joint replacement procedures [1] but its use has now extended to involve pharmacological treatment, physiotherapy and fractures [2]. OKS versions presently exist in many different languages, with 4 approved Chinese versions, including

\footnotetext{
*Correspondence: zipromax@163.com

${ }^{1}$ School of Clinical Medicine, Tsinghua University, Beijing 100084, China

${ }^{2}$ Tsinghua University Haidian district, Zijing Apartment 21, Beijing 100084,

China

Full list of author information is available at the end of the article
}

OKS Malaysian Chinese, OKS Hong Kong Cantonese and OKS Singapore Chinese versions, which were all validated in their respective regions [3, 4] and a Mainland Chinese OKS version [5]. We conducted a systematic online database search in PubMed, Embase, Web of Science, China Knowledge Resource Integrated Database (CNKI), WeiPu database (VIP) and WanFangData on the use of OKS scores in Mainland China, with time frame ranging from inception up to 2017. In addition to the aforementioned versions, this search revealed 3 more OKS Chinese versions in use. It is imperative to use high-quality validated questionnaires since poorlydesigned questionnaires can potentially lead to

(c) The Author(s). 2020 Open Access This article is licensed under a Creative Commons Attribution 4.0 International License, which permits use, sharing, adaptation, distribution and reproduction in any medium or format, as long as you give appropriate credit to the original author(s) and the source, provide a link to the Creative Commons licence, and indicate if changes were made. The images or other third party material in this article are included in the article's Creative Commons licence, unless indicated otherwise in a credit line to the material. If material is not included in the article's Creative Commons licence and your intended use is not permitted by statutory regulation or exceeds the permitted use, you will need to obtain permission directly from the copyright holder. To view a copy of this licence, visit http://creativecommons.org/licenses/by/4.0/. 
unreliable and misleading results [6]. The English language OKS versions in use in different countries are very similar, but there are differences in expressions and use of words among various Chinese versions. Moreover, the OKS Hong Kong version uses traditional Cantonese characters. The aim of the study was to carry out validation and reliability studies of the 3 non-mainland Chinese versions to ascertain their interchangeable usage in mainland China.

\section{Materials and methods}

The Oxford Knee Score (OKS) has 12-items, each having 5 ordinal response options over a recall period "during the last 4 weeks." Each question is scored on a 0-to-4 point scale with 4 being the best outcome and the overall scores range from 0 to 48 [2].

Permission and licence for the use of OKS scores were granted by Oxford Innovation. Smart device-compatible electronic versions of all the scores were designed according to recommendations from Oxford Innovation and approved for use in the study. An electronic version of the SF-36 was also designed for patient completion.

WeChat ${ }^{\circ}$ (Tencent, Shenzhen, China) application is a widely used social media platform in Mainland China, available over a wide variety of smart devices. The electronic versions were designed using online software tools which were compatible with the $\mathrm{WeChat}^{\circ}$ platform. This study was approved by the Clinical Research Ethics Committee of our institution.

\section{Patients}

One hundred ninety-four patients participated in the study, and were recruited over a period of 4 months from the General Orthopaedic Outpatient Clinic and Arthroplasty Specialists Clinic of our level-3 general hospital in Beijing. Participants were diagnosed with knee osteoarthritis on the basis of clinical symptoms and signs and the presence of positive radiographic findings as confirmed by two orthopaedic surgeons. The inclusion criteria were: (1) an ability to read and write Chinese, (2) having been diagnosed with knee osteoarthritis, (3) having access to and being able to use WeChat ${ }^{\circ}$ platform. Participants were either newly diagnosed or had new onset of symptoms following a previous diagnosis. Patients on ongoing treatment were not included. Participants' demographics, including gender, age and knee side involved, are listed in Table 1.

Participants were randomly assigned into 3 groups. Group A (OKS Malaysia Chinese) had 63 patients, group B (OKS Singapore Chinese) consisted of 67 patients and group C (OKS Hong Kong Cantonese) was comprised of 64 patients. Sample sizes referred to recommendations from previous studies that at least 50 subjects should be included for comparison studies [7, 8] (Table 1).
Table 1 Participants' demographics

\begin{tabular}{llll}
\hline Characteristics & $\begin{array}{l}\text { Group A } \\
(\boldsymbol{n}=\mathbf{6 3})\end{array}$ & $\begin{array}{l}\text { Group B } \\
(\boldsymbol{n}=\mathbf{6 7})\end{array}$ & $\begin{array}{l}\text { Group C } \\
(\boldsymbol{n}=64)\end{array}$ \\
\hline Gender & & & \\
$\quad$ Female & 37 & 29 & 39 \\
$\quad$ Male & 26 & 38 & 25 \\
Knee Side & & & \\
$\quad$ Left & 19 & 23 & 21 \\
Right & 20 & 23 & 22 \\
Both & 24 & 21 & 21 \\
Mean (SD) Age (years) & $48.16(12.79)$ & $51.58(12.89)$ & $47.27(12.69)$
\end{tabular}

SD Standard Deviation

The participants received the questionnaires via smart devices after consultation and were briefly instructed on how to complete the forms. During the form completion, the patients were asked to point out any difficulties in language comprehension of questions of the Score. During the process, questions were randomly selected and patients asked to explain the content. Participants were sent OKS questionnaires a second time via $\mathrm{WeChat}^{\circ}$ platform for reliability analysis.

\section{Psychometrics}

To asses test and retest reliability, patient responses of the first and second OKS measurements were compared. Previous studies used a time frame of 1-14 days between the completion of the scores, during which a patient's clinical status is unlikely to experience any major changes in the absence of specific intervention $[1,9]$. Test-retest reliability was calculated using the intraclass correlation coefficient (ICC) to evaluate reproducibility. Cronbach's $\alpha$ coefficient was used to measure internal consistency.

The construct validity was examined by means of convergent validity and divergent validity. The OKS scores were compared to the various domains of the SF-36 by calculating the Spearman's rank correlation coefficients ( $\rho$ ). The SF 36 assesses health under two headings: Physical Component Summary (PCS), which includes Physical Functioning (PF), Role Physical (RP), Bodily Pain (BP) and General Health (GH) and Mental Component Summary (MCS), which involves Vitality (VT), Social Functioning (SF), Role Emotional (RE) and Mental Health (MH). According to studies from Juniper et al, correlation values of $>0.50,0.35$ to 0.50 , and $<0.35$ can be interpreted as having strong, moderate, and weak correlation, respectively [10]. On the basis of information, we hypothesized there are strong correlations between OKS and related domain scores of the SF- 36 and weak correlations with non-related domains on the SF-36.

The statistical analysis was performed using $\mathrm{SPSS}^{\circ}$ Version 20.0 software package (SPSS Inc., Chicago, IL, USA). 


\section{Results}

\section{Reliability studies}

The OKS was completed a second time by 38 patients from Group A with an average time 3.26 (1.79) days from the first completion; 35 patients in Group B with an average time of 2.69 (1.07) days; 38 patients from Group $\mathrm{C}$ with an average time of 3.08 (1.17) days. The test-retest reliability calculated with ICC in all $3 \mathrm{OKS}$ groups was $0.917,0.921$, and 0.824 respectively (Table 2 ). The Cronbach's $\alpha$ coefficient for the 3 groups was 0.912 , 0.896 and 0.846 , respectively (Table 3 ).

\section{Validity studies}

Group A showed strong negative correlations with related domains of the SF-36, bodily pain $(\rho=-0.724, \rho<$ 0.001 ) and physical functioning ( $\rho=-0.538, p<0.001)$. Group A showed weak correlations with unrelated domains: vitality, role emotional and mental health but these correlations were not significant $(\rho>0.05)$. Group $B$ exhibited moderate correlations with related domains of the SF-36, bodily pain ( $\rho=-0.495, p<0.001)$, physical function $(p=-0.406, p<0.001)$. Group B bore weak correlations with unrelated domains: vitality, role emotional and mental health but these were not significant $(p>$ $0.05)$. Group $C$ reveled strong negative correlations with physical functioning $(\rho=-0.655, p<0.001)$, bodily pain ( $\rho=-0.565, p<0.001$, and weak negative correlations with unrelated domains vitality $(\rho=-0.311, p<0.05)$. Correlations with mental health and role emotional were not significant (Table 4).

\section{Discussion}

Currently, there are four different versions of the Chinese $\mathrm{OKS}$, and all have been validated in sample populations in their various regions. Knee-related studies in mainland China using mainland-based samples employed various Chinese OKS versions. Beaton et al studied the crosscultural adaptation guidelines and suggested that the same language questionnaires used in different countries have to undergo cross-cultural adaptation and psychometric analysis [11]. So, this study analyzed the psychometric properties of the available Chinese OKS versions in a mainland sample population so as to demonstrate their feasibility for use in Mainland-based studies.

Test-retest reliability calculation in the different groups showed excellent results, with ICC $>0.8$ and accepted threshold $\geq 0.75$ [12]. Internal consistency test in

Table 2 Test and retest reproducibility for 3 OKS versions

\begin{tabular}{llll}
\hline OKS Version & ICC & $\mathbf{9 5 \% ~ C l}$ & $\boldsymbol{p}$ value \\
\hline Group A & 0.917 & $0.841-0.957$ & $<0.001$ \\
Group B & 0.921 & $0.844-0.960$ & $<0.001$ \\
Group C & 0.824 & $0.666-0.910$ & $<0.001$ \\
\hline
\end{tabular}

Table 3 Internal consistency for 3 different OKS versions

\begin{tabular}{ll}
\hline OKS version & Cronbach's alpha \\
\hline Group A & 0.912 \\
Group B & 0.896 \\
Group C & 0.846 \\
\hline
\end{tabular}

terms of Cronbach's $\alpha$ yielded equally good results, with ICC $>0.80$ and accepted threshold $\geq 0.7$ [13]. Reliability results were similar to those obtained on the different OKS versions in their respective geographical settings, with all $>0.80$.

In this study, construct validity was assessed by using only SF-36. Factor analysis of previous studies demonstrated that OKS could be composed of 2 factors, i.e., knee pain and knee dysfunction [9]. We thus expected strong correlations with similar related domains of the SF-36. The present study indicated a convergent validity. Results showed correlations between similar domains in the OKS, with moderate to strong correlations found with bodily pain and physical function, which was consistent with our hypothesis. Validation studies from the sample study in Singapore showed strong negative correlation with the Physical Functioning domain [4]. Strongest correlations in validation studies from the Hong Kong sample were with Physical Functioning and Bodily Pain domains [3]. The mainland Chinese version that used Pearson correlation coefficient for validation studies revealed moderate correlations with both the physical component summary of the SF-36 as well as the mental component summary [5].

The present Study utilized electronic from versions instead of the traditional paper-based formats, and the overall feedback from the participants was acceptable.

Table 4 Spearman correlation between different OKS groups and SF-36

\begin{tabular}{cccc}
\hline SF-36 & Group A & Group B & Group C \\
\hline PCS & & & \\
PF & $-0.538^{\mathrm{a}}$ & $-0.406^{\mathrm{a}}$ & $-0.655^{\mathrm{a}}$ \\
RP & $-0.479^{\mathrm{a}}$ & -0.198 & -0.263 \\
BP & $-0.724^{\mathrm{a}}$ & $-0.495^{\mathrm{a}}$ & $-0.565^{\mathrm{a}}$ \\
GH & $-0.325^{\mathrm{b}}$ & -0.123 & $-0.426^{\mathrm{a}}$ \\
MCS & & & \\
VT & -0.050 & -0.107 & $-0.311^{\mathrm{b}}$ \\
SF & $-0.328^{\mathrm{b}}$ & $-0.438^{\mathrm{a}}$ & $-0.353^{\mathrm{b}}$ \\
RE & -0.097 & -0.101 & -0.272 \\
MH & 0.189 & -0.050 & -0.286 \\
\hline
\end{tabular}

${ }^{a}$ Correlation is significant at the 0.01 level (2-tailed), ${ }^{\mathrm{b}}$ Correlation is significant at the 0.05 level (2-tailed), MCS Mental Component Summary, PCS Physical Component Summary

PF Physical Functioning, RP Role Physical, BP Bodily Pain, GH General Health, MCS Mental Component Summary, including $V T$ Vitality, SF Social Functioning, $R E$ Role Emotional and $M H$ Mental Health 
Patient Reported Outcome Measures (PROMs) primarily have to be completed by the patients and 'e-PROMs' provide a relatively inexpensive and fast way of participants' follow-up, and are especially useful for patients seeking consultation from other cities, a common phenomenon in large hospitals in tier-1 cities. Previous studies have shown patients' preference in the use ePROMs, with their advantages including convenience and economy in terms of time, manpower and money. Errors due to manual transfer of paper data to electronic devices can also be avoided by using e-PROMs [14]. Results from meta-analysis on 65 studies conducted by Gwaltney CJ et al strongly suggested an equivalence between paper-based and e-PROMs [15]. The design of ePROMs also has to follow strict guidelines, which stipulate that moderate and substantial modifications, including changing the wordings of the items or response options, should have evidence through equivalence studies and full psychometric testing. The e-PROMs used in this study involved only minor modifications and did not require psychometric studies prior to use [16]. However, patients brought up the issue of privacy and protection of information, which is a major security concern with smart devices. No personal or private information was collected and patients could provide their initials rather than full names. In administering e-PROMs and building databases, healthcare institutions need to scrupulously protect the systems to ensure the security of patient data.

\section{Conclusion}

Different Chinese versions of the OKS were proven to be reliable and valid in mainland samples of patients with knee osteoarthritis, thereby supporting their use in research and other related studies.

\section{Abbreviations \\ OKS: Oxford Knee Score; SF 36: 36-Item Short Form Survey; CNKI: China Knowledge Resource Integrated Database; VIP: WeiPu database; ICC: Intraclass correlation coefficient; PROMs: Patient Reported Outcome Measures}

\section{Acknowledgements}

We would like to acknowledge The Oxford University Innovation Limited, for their kind support.

\section{Authors' contributions}

Ngwayi James Reeves Mbori: Study design, outpatient data collection, data analysis, paper writing. Tan Jie: Electronic Prom design and data collection. Liang Ning: Outpatient participant recruitment, data collection. Daniel Edward Porter: Study design, obtaining license for use of OKS Prom, study coordination, proof reading. The authors read and approved the final manuscript.

\section{Funding}

This study received no funding support.

\section{Availability of data and materials}

The datasets generated during the current study are not publicly available due to the fact that some data sets contain participants' personal information, such as names and phone numbers.
Ethics approval and consent to participate

The project was approved by Beijing HuaXin Hospital Ethics Committee (Reference number: 2020-02).

Consent for publication

Not applicable.

\section{Competing interests}

The authors declare that they have no competing interests.

\section{Author details}

${ }^{1}$ School of Clinical Medicine, Tsinghua University, Beijing 100084, China. ${ }^{2}$ Tsinghua University Haidian district, Zijing Apartment 21, Beijing 100084 China. ${ }^{3}$ Department of Orthopedics, Beijing Huaxin Hospital, Clinical Medicine School, Tsinghua University, Beijing 100016, China.

Received: 4 June 2020 Accepted: 4 October 2020

Published online: 21 October 2020

\section{References}

1. Dawson J, Fitzpatrick R, Murray D, Carr A. Questionnaire on the perceptions of patients about total knee replacement. J Bone Joint Surg Br. 1998;80:63-9.

2. Murray DW, Fitzpatrick R, Rogers K, Pandit H, Beard DJ, Carr AJ, et al. The use of the Oxford hip and knee scores. J Bone Joint Surg Br. 2007;89-B:1010-4.

3. Cheung RTH, Ngai SPC, Ho KKW. Chinese translation and validation of the Oxford knee scale for patients with knee osteoarthritis. Hong Kong Physiother J. 2017;37:46-9.

4. Xie F, Li S-C, Roos EM, Fong K-Y, Lo N-N, Yeo S-J, et al. Cross-Cultural adaptation and validation of Singapore English and Chinese versions of the Oxford knee score (OKS) in knee osteoarthritis patients undergoing total knee replacement. Osteo Arthritis and Cartilage. 2007;15:1019-24.

5. Kai Lin BS, Liangxiao Bao BS, Jian Wang MD, Kimie Fujita PD, Kiyoko Makimoto PD, Xiaoyan Liao PD. Validation of the Chinese (mandarin) version of the Oxford knee score in patients with knee osteoarthritis. Clin Orthop Relat Res. 2017;475:2992-3004.

6. Gagnier JJ. Patient reported outcomes in orthopaedics. J Orthop Res. 2017; 35:2098-108.

7. Altman DG. Practical statistics for medical research. London: Chapman and Hall; 1991. p. 611.

8. Ekeberg OM, Bautz-Holter E, Tveita EK, Keller A, Juel NG, Brox JI. Agreement, reliability and validity in 3 shoulder questionnaires in patients with rotator cuff disease. BMC Musculoskelet Disord. 2008;9:68.

9. Eun IS, Kim OG, Kim CK, Lee HS, Lee JS. Validation of the Korean version of the Oxford knee score in patients undergoing Total knee Arthroplasty. Clin Orthop Relat Res. 2013;471:600-5.

10. Juniper EF, Gordon HG, Roman J. How to develop and validate a new health-related quality of life instrument. In: Spilker B, editor. Quality of life and Pharmacoeconomics in clinical trials. 2nd ed. Philadelphia: LippincottRaven Publishers; 1996. p. 49-56.

11. Beaton DE, Bombardier C, Guillemin F, Ferraz MB. Guidelines for the process of cross-cultural adaptation of self-report measures. Spine (Phila Pa 1976). 2000;25:3186-91.

12. Rosner B. Fundamentals of biostatistics. 4th ed. Belmont: Duxbury Press; 1995.

13. Cronbach $\perp$. Coefficient alpha and the internal structure of tests. Psychometrika. 1951;16:297-334.

14. Campbell N, Ali F, Finlay AY, Salek SS. Equivalence of electronic and paperbased patient-reported outcome measures. Qual Life Res. 2015;24:1949-61.

15. Gwaltney CJ, Shields AL, Shiffman S. Equivalence of electronic and paperand-pencil Administration of Patient-Reported Outcome Measures: a metaanalytic review. Value Health. 2008;11(2):322-33.

16. Patient Reported Outcomes -From Paper to ePROs: Good Practice Guide for Migration. The Clinical Outcomes team at Oxford University Innovation 2016.

\section{Publisher's Note}

Springer Nature remains neutral with regard to jurisdictional claims in published maps and institutional affiliations. 